\title{
Immunological measurement of aspartate/alanine aminotransferase in predicting liver fibrosis and inflammation
}

Hyun Jeong Kim 1, ${ }^{\star}$, Sang Yeol Kim ${ }^{2,}$, Suk Pyo Shin², Young Joo Yang ${ }^{2}$, Chang Seok Bang ${ }^{2}$, Gwang Ho Baik², Dong Joon $\mathrm{Kim}^{2}$, Young Lim $\mathrm{Ham}^{3}$, Eui Yul $\mathrm{Choi}^{1}$, and Ki Tae Suk ${ }^{2}$

${ }^{1}$ Boditech Central Research Institute, Chuncheon; ${ }^{2}$ Department of Internal Medicine, Hallym University College of Medicine, Chuncheon; ${ }^{3}$ Department of Emergency Medical Technology, Daewon University College, Jecheon, Korea
Received: June 19, 2018 Revised : October 1, 2018 Accepted: October 1, 2018

\section{Correspondence to}

Ki Tae Suk, M.D.

Department of Internal Medicine, Hallym University Chuncheon Sacred Heart Hospital, 77 Sakju-ro, Chuncheon 24253, Korea

Tel: +82-33-240-5826

Fax: +82-33-241-8064

E-mail: ktsuk@hallym.ac.kr

*These authors contributed equally to this work.
Background/Aims: Enzymatic analysis of aspartate/alanine aminotransferase (AST/ALT) does not exactly represent the progression of liver fibrosis or inflammation. Immunoassay for AST (cytoplasmic [c] AST/mitochondrial [m] AST) and ALT (ALT1/ALT2) has been suggested as one alternatives for enzymatic analysis. The objective of this study was to evaluate the efficacy of immunoassay in predicting liver fibrosis and inflammation.

Methods: A total of 219 patients with chronic hepatitis B (CHB) who underwent hepatic venous pressure gradient (HVPG) and liver biopsy before antiviral therapy were recruited. Serum samples were prepared from blood during HVPG. Results of biochemical parameters including enzymatic AST/ALT and immunological assays of cAST, mAST, ALT1, and ALT2 through sandwich enzyme-linked immunosorbent assay (ELISA) immunoassay with fluorescence labeled monoclonal antibodies were compared with the results of METAVIR stage of live fibrosis and the Knodell grade of inflammation.

Results: METAVIR fibrosis stages were as follows: Fo, six (3\%); F1, 52 (24\%); F2, 88 (40\%); F3, 45 (20\%); and F4, 28 patients (13\%). Mean levels of AST and ALT were 121 \pm 157 and $210 \pm 279 \mathrm{IU} / \mathrm{L}$, respectively. Mean HVPG score of all patients was $4.7 \pm 2.5$ $\mathrm{mmHg}$. According to the stage of liver fibrosis, HVPG score $(p<0.001, r=0.439)$ and ALT1 level $(p<0.001, r=0.283)$ were significantly increased in all samples from patients with CHB. ALT $(p<0.001, r=0.310), \operatorname{ALT1}(p<0.001, r=0.369)$, and $\operatorname{AST}(p<0.001, r=0.374)$ levels were positively correlated with Knodell grade of inflammation.

Conclusions: ALT1 measurement by utilizing sandwich ELISA immunoassay can be useful method for predicting inflammation grade and fibrosis stage in patients with CHB.

Keywords: Alanine aminotransferase; Aspartate transferase; Enzyme-linked immunosorbent assay; Hepatitis B, chronic

\section{INTRODUCTION}

Aspartate aminotransferase (AST) and alanine amino- transferase (ALT) participate in intermediary metabolism and in liver gluconeogenesis [1]. AST can be detected in the liver, heart, muscle, kidneys, brain, and blood 
cells. ALT is found in plasma and other body organs, although it is most commonly found in the liver. AST and ALT are released by liver tissue into circulation in proportion to the degree of hepatocellular damage due to toxic substances, viral infections, or other causes of liver damage [2]. In most types of liver disease, serum ALT exhibits greater enzymatic activity than serum AST [3]. In clinical laboratories, serum AST and ALT activities are commonly measured by enzymatic assay in which transamination reaction is coupled to a secondary reaction that reduces pyruvate into lactate via lactate dehydrogenase [4].

The development of an accurate diagnostic method is critical for early detection and proper treatment of liver disease, because patients are often asymptomatic until their livers have deteriorated severely [5]. Most liver diseases can be diagnosed by enzymatic method for serum AST and ALT [6,7]. However, the enzymatic method does not represent the state of liver fibrosis. It can cause misdiagnosis in patients with chronic liver diseases such as fatty liver disease, cirrhosis, and hepatocellular carcinoma [8]. Although the definition of chronic hepatic injury by increased ALT is widely accepted, most patients with liver cirrhosis (LC) have persistently normal or mild elevated ALT.

Earlier studies have indicated there are two isoforms of ALT. ALT1 is mainly expressed in the liver and kidney while ALT2 is mainly expressed in the pancreas, brain, adrenal gland, skeletal muscle, and heart [9]. Two types of AST (isoenzymes) have also been found in animal tissues, one in the mitochondria (mAST) and the other in the cytosol matrix (cAST) [10]. Although they differ markedly in primary structure and chemical property, both mAST and cAST can catalyze the same reaction with subtly different catalytic steps [11,12].

Previous reports have suggested that ALT-immunoglobulin complex increased according to the severity of liver disease, and that high concentration of mAST might indicate a severely damaged liver $[4,13]$. Therefore, immunoassay might be useful as a screening method for differential diagnosis of liver fibrosis according to patients. The objective of the present study was to determine the efficacy of immunoassay in predicting liver fibrosis and inflammation in patients with chronic hepatitis $\mathrm{B}(\mathrm{CHB})$.

\section{METHODS}

\section{Patients and diagnosis}

Between January 2007 and December 2010, 271 patients with $\mathrm{CHB}$, who simultaneously underwent hepatic venous pressure gradient (HVPG) and liver biopsy for the routine check-up prior to antiviral treatment, were prospectively recruited. Patients were selected according to inclusion criteria with CHB. Some of them were then excluded according to following exclusion criteria: (1) those with other cause of liver disease; (2) those with decompensated cirrhosis; or those who received antiviral treatment within the previous 6 months. Fifty-two patients with evidence of decompensated LC such as HVPG $>10 \mathrm{mmHg}$, ascites, or varix were excluded. Finally, a total of 219 patients with CHB were enrolled in the study. Their baseline characteristics are summarized in Table 1. None of these CHB patients had evidence of LC at initial time, verified by abdominal ultrasound, endoscopy, or neurological examination. The diagnosis of LC was established by a liver biopsy and/or imaging studies such as ultrasound and/or contrast-enhanced computed tomography in conjunction with laboratory results and clinical complications of cirrhosis (presence of ascites, hepatic encephalopathy, and esophageal varices). Decompensated LC was diagnosed according to the current definition in the presence of ascites, portal hypertensive gastrointestinal bleeding, encephalopathy, or jaundice $[14,15]$.

Protocols for this study conform to the ethical guidelines established by the 2013 Declaration of Helsinki as reflected in a priori approval by the Institutional Review Board for Human Research (2007-02). Informed consent to participate in the study was obtained from each patient (ClinicalTrials.gov Identifier: NCT03562585)(Fig. 1).

Researchers of this study conducted baseline evaluations which included family and alcohol history, $\mathrm{X}$-ray, electrocardiography, electrolyte, liver function test, complete blood count, and virus markers. Serum biochemical parameters included total bilirubin, ALT, haptoglobin, AST, international normalized ratio (INR), albumin, blood urea nitrogen, creatinine, gamma glutamyl transpeptidase, alkaline phosphatase, $\alpha$-fetoprotein, prothrombin time, blood glucose, triglycerides, and total cholesterol. Markers of hepatitis virus (hepatitis B virus surface antigen [HBsAg], hepatitis B surface 
Table 1. Baseline patient characteristics

\begin{tabular}{|c|c|}
\hline Variable & All patients $(\mathrm{n}=219)$ \\
\hline Male sex & $146(67)$ \\
\hline Age, yr & $39.7 \pm 11.3$ \\
\hline Hemoglobin, g/dL & $14.3 \pm 1.7$ \\
\hline Platelets, $\times 10^{3} / \mathrm{mm}^{3}$ & $207 \pm 67$ \\
\hline International normalized ratio & $1.6 \pm 7.2$ \\
\hline Total bilirubin, mg/dL & $0.9 \pm 1.2$ \\
\hline Albumin, g/dL & $4.1 \pm 0.4$ \\
\hline $\mathrm{AST}, \mathrm{IU} / \mathrm{L}^{\mathrm{a}}$ & $121 \pm 157$ \\
\hline $\mathrm{ALT}, \mathrm{IU} / \mathrm{L}^{\mathrm{a}}$ & $210 \pm 279$ \\
\hline HBV DNA, × $10^{5} \mathrm{IU} / \mathrm{mL}$ & $200 \pm 391$ \\
\hline HBeAg positive & $135(63)$ \\
\hline HVPG score, mmHg & $4.7 \pm 2.5$ \\
\hline \multicolumn{2}{|l|}{ METAVIR stage } \\
\hline Fo & $6(3)$ \\
\hline $\mathrm{F}_{1}$ & $52(24)$ \\
\hline $\mathrm{F} 2$ & $88(40)$ \\
\hline $\mathrm{F}_{3}$ & $45(20)$ \\
\hline $\mathrm{F}_{4}$ & $28(13)$ \\
\hline \multicolumn{2}{|l|}{ Knodell grade } \\
\hline Go & $15(7)$ \\
\hline G1 & $62(28)$ \\
\hline G2 & $82(38)$ \\
\hline G3 & $57(26)$ \\
\hline $\mathrm{G}_{4}$ & $3(1)$ \\
\hline
\end{tabular}

Values are presented as number (\%) or mean \pm SD.

AST, aspartate aminotransferase; ALT, alanine aminotransferase; HBV, hepatitis B virus; $\mathrm{HBeAg}$, hepatitis B e antigen; HVPG, hepatic venous pressure gradient.

${ }^{\mathrm{a} E n z y m a t i c}$ analysis.

antibody [anti-HBs], Hepatitis B e antibody [anti-HBe], hepatitis B virus [HBV] DNA, hepatitis B core antibody [anti-HBc], hepatitis B e antigen [HBeAg], anti-hepatitis $\mathrm{C}$ virus [HCV], and HCV RNA) were also checked.

\section{HVPG measurement}

HVPG measurement was performed by two hepatologists (K.T.S.). A 6 French balloon catheter was placed in the right hepatic venous (HV) through a right jugular vein puncture to measure free HV pressure. Wedged hepatic venous pressure (WHVP) was measured by in-

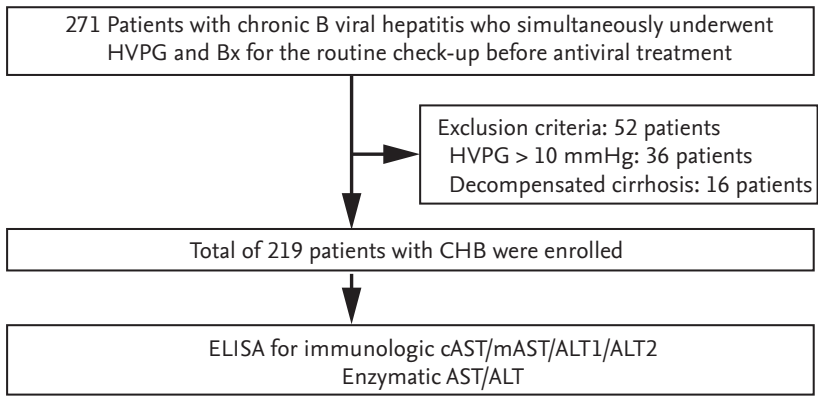

Figure 1. Study design. HVPG, hepatic venous pressure gradient; Bx, biopsy; CHB, chronic hepatitis B; ELISA, enzyme-linked immunosorbent assay; cAST, cytoplasmic aspartate aminotransferase; $\mathrm{mAST}$, mitochondrial aspartate aminotransferase; ALT, alanine aminotransferase.

flating the balloon catheter at the right $\mathrm{HV}$ or middle hepatic vein. Subsequently, HVPG was determined by subtracting the free HV pressure from the WHVP. The procedure allowed at least 1 minute for the WHVP and 15 seconds for free HV pressure stabilization. The average was taken from three separate readings. For cases with the presence of a shunt, the measurement was taken at a different location to minimize error [16].

\section{Sandwich enzyme-linked immunosorbent assay}

A sandwich enzyme-linked immunosorbent assay (ELISA) system was used to measure enzymatic activity of ALT, AST, and immunologic activity of ALT1, ALT2, mAST, cAST using serum samples. A 96-well micro-plate was coated with capture antibodies in coating buffer $(100 \mu \mathrm{L} /$ well) and subsequently incubated at $4{ }^{\circ} \mathrm{C}$ overnight. After washing with phosphate buffered saline-Tween (PBS-T) $0.05 \%$ three times, plates were blocked with $5 \%$ skimmed milk in PBS-T ( $200 \mu \mathrm{L} /$ well) at $37^{\circ} \mathrm{C}$ for 2 hours. After another washing step, a $100 \mu \mathrm{L}$ sample solution was added to each well, and the microplates were incubated at $37^{\circ} \mathrm{C}$ for 1 hour. After $100 \mu \mathrm{L}$ of horseradish peroxidase-labeled anti-ALT, ALT1, ALT2, AST, cAST, or mAST antibody was added to each well, these micro-plates were incubated at $37^{\circ} \mathrm{C}$ for 1 hour. After washing three times, 3,3',5,5'-tetramethylbenzidine (TMB) substrate solution was added to each well and reacted at $37^{\circ} \mathrm{C}$ for $15 \mathrm{~min}$ utes in the dark. The reaction was stopped by adding 2 M sulfuric acid (50 $\mu \mathrm{L} /$ well). Absorbance was measured at wavelength of $450 \mathrm{~nm}$. All samples were processed in duplicates. 


\section{Histology}

A percutaneous liver biopsy (ultrasound guided) was performed using an 18-gauge needle. Biopsy specimens were fixed with $10 \%$ formalin, routinely embedded in paraffin, and sectioned. These sections were processed for hematoxylin and eosin, Masson's trichrome, or reticulin fiber staining. Necroinflammatory change and liver fibrosis of all biopsy specimens were evaluated using Knodell grade and METAVIR scoring system, respectively. The Knodell grade includes three subcategories: periportal necrosis and inflammation, scored from o to 10; intralobular necrosis and inflammation, scored from o to 4; and portal inflammation, scored from o to 4 $[17,18]$. Fibrosis was scored from o to 4 as follows: Fo, no fibrosis; F1, enlarged fibrotic portal tracts; F2, enlargement of portal tracts with rare periportal or portal-portal septa; F3, numerous septa without cirrhosis; and F4, cirrhosis $[17,19]$.

\section{Statistical analysis}

Quantitative data were expressed as mean \pm standard deviation unless otherwise stated. The correlation of HVPG and liver biopsy results was evaluated using Pearson bivariate correlations analysis and expressed box plot. The diagnostic difference between HVPG and biopsy was evaluated by McNemar's test (matched-pair data) [20]. Age, gender, and some risk factors $(p<0.10)$ identified by the univariate analysis were entered into multivariate analysis using stepwise forward selection. Results from these analyses were used to develop a model for the diagnosis of stage 1 compensated LC based on findings of HVPG alone, biopsy alone, and both HVPG and biopsy. All statistical tests were two sided and performed with SPSS software version 19.0 (IBM Co., Armonk, NY, USA). For all tests, a $p$ value of less than 0.05 was considered to be statistically significant.

\section{RESULTS}

\section{Patient characterization}

The mean age of all patients was $39.7 \pm 11.3$ years. The mean levels of AST and ALT were $121 \pm 157$ and $210 \pm$ 279 IU/L, respectively. The average HVPG score for all patients was $4.7 \pm 2.5 \mathrm{mmHg}$. Twelve biopsy specimens (5.4\%) showed discordance in pathology. A consensus

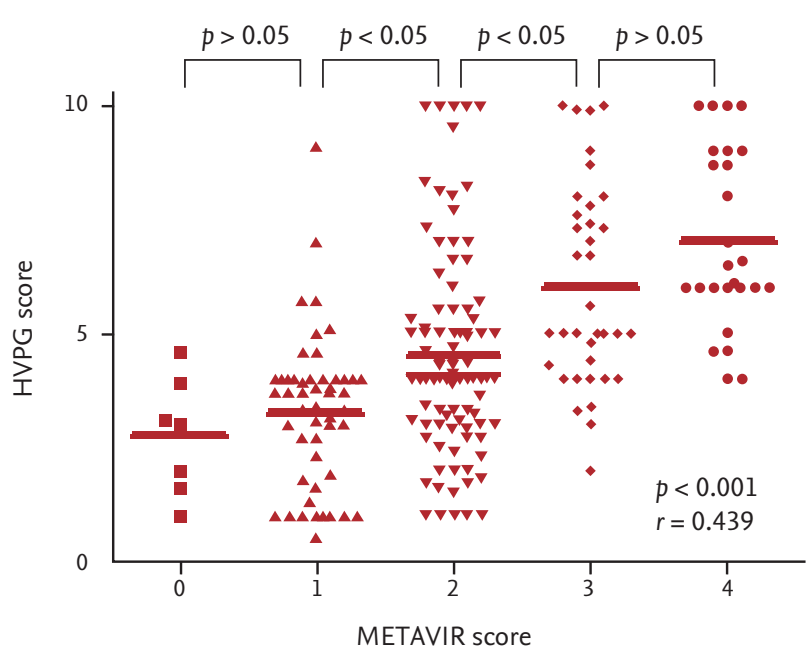

Figure 2. Hepatic venous pressure gradient (HVPG) score according to METAVIR score.

was reached through discussions for cases showing discordance. The distribution of the METAVIR fibrosis stages were as follows: Fo, six (3\%); F1, 52 (24\%); F2, 88 (40\%); F3, 45 (20\%); and $\mathrm{F}_{4}, 28$ patients (13\%). The distribution of Knodell inflammation grade were: Go, 15 (7\%); G1, 62 (28\%); G2, 82 (38\%); G3, 57 (26\%); and G4, 3 (1\%) (Table 1).

Patients with LC on HVPG and biopsy were significantly older than those in other groups. Platelet count and albumin levels were remarkably higher in patients with LC diagnosed through HVPG alone compared to those in patients with LC diagnosed with liver biopsy alone. There were no significant differences in other variables between the two groups of patients $(p>0.05)$. In the analysis of the HVPG score according to ALT lev$\mathrm{el}$, the ALT level did not affect the HVPG score $(p>0.05)$.

\section{HVPG score according to METAVIR fibrosis stage}

The mean level of HVPG score was positively correlated with the METAVIR fibrosis score $(p<0.001, r=0.439)$ (Fig. 1). There were significant stepwise differences among METAVIR stage 1, 2, and 3. However, there were no significant differences between METAVIR stage $O$ and stage 1 . This result was seen in the relationship between METAVIR stages 3 and 4 ( $p>0.05$ ). Fifty-seven and 28 patients were diagnosed as stage 1 compensated LC through HVPG and biopsy, respectively. Twenty patients were diagnosed as stage 1 compensated LC di- 

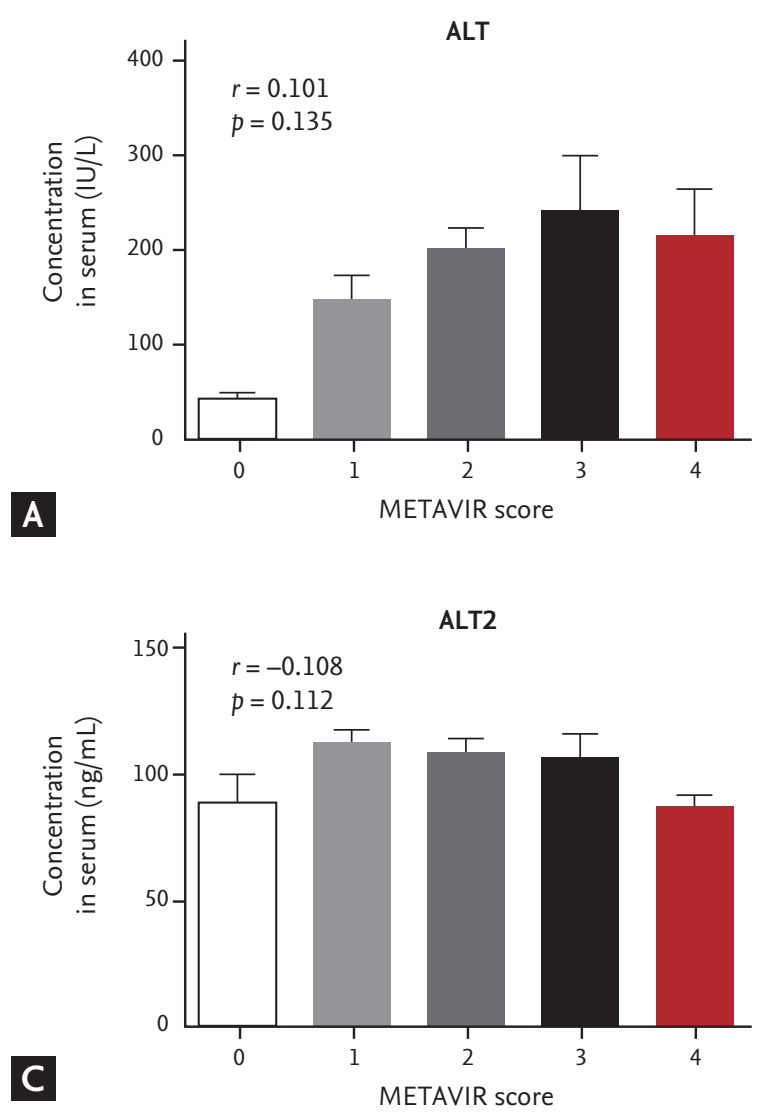
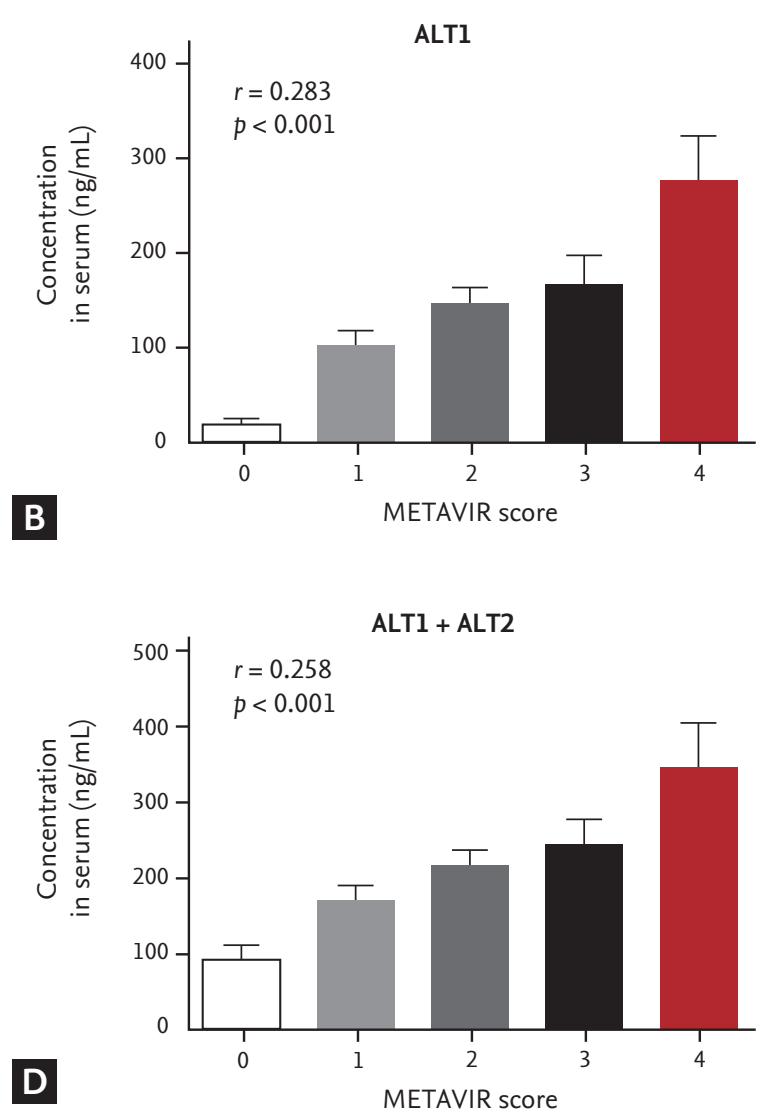

Figure 3. Distribution of alanine aminotransferase (ALT) mass concentration with METAVIR score. Serum samples were measured side by side for ALT concentration with an immunoassay. (A) Immunoassay of enzymatic ALT with METAVIR score. (B) Immunoassay of immunologic ALT1 with METAVIR score. (C) Immunoassay of immunologic ALT2 with METAVIR score. (D) Immunoassay of immunologic ALT1 + ALT2 with METAVIR score.

agnosed with both HVPG and biopsy. In general, there were significant differences between the two methods for the diagnosis of stage 1 compensated LC in patients with $\mathrm{CHB}(p<0.01)$ (Fig. 2).

\section{Correlation between ALT level and METAVIR stage}

Mean levels of ALT (IU/L) according to fibrosis stage were Fo (41.2), F1 (149.9), F2 (200.4), F3 (242.8), and F4 (214.1) in each fibrosis stage $(p=0.135, r=0.101)$, showing no significant correlation. ALT1 levels $(\mathrm{ng} / \mathrm{mL})$ according to fibrosis stage were Fo (19.6), F1 (101.5), F2 (147.2), F3 (165.6), and $\mathrm{F}_{4}$ (277.8), showing significant correlation $(p<$ $0.001, r=0.283)$. ALT2 levels $(\mathrm{ng} / \mathrm{mL})$ according to fibrosis stage were Fo (90.1), F1 (112.3), F2 (109.2), F3 (106.5), and $\mathrm{F}_{4}$ (86.8), showing no significant correlation $(p=0.112, r$ $=0.108)$. Mean level of ALT1 + ALT2 (ng/mL) according to fibrosis stage were Fo (91.9), F1 (169.3), F2 (216.0), F3
(242.5), and $\mathrm{F}_{4}$ (349.8), showing significant correlation $(p<$ $0.001, r=0.258)$. The ALT + ALT1 + ALT2 in each fibrosis stage were Fo (150.8), F1 (360.8), F2 (456.7), F3 (514.9), and $\mathrm{F}_{4}$ (578.6), showing significant correlation $(p=0.001, r=$ 0.216). Although ALT or ALT2 did not show significant correlation with METAVIR stages, ALT1, ALT1 + ALT2, and ALT + ALT1 + ALT2 were significantly related with the METAVIR stage (Fig. 3).

\section{Correlation between ALT level and Knodell grade}

For inflammation grades Go, G1, G2, G3, and $\mathrm{G}_{4}$, mean levels of ALT (IU/L) were 75.2, 126.9, 197.6, 261.6, and 911, respectively, showing significant correlation $(p<0.001$, $r=0.310)$. ALT1 levels (ng/mL) according to Knodell grade were Go (54.5), G1 (94.5), G2 (142.1), G3 (237.2), and $\mathrm{G}_{4}$ (549.9), showing significant correlation $(p<0.001, r=$ 0.369). ALT2 levels (ng/mL) according to inflammation 
ALT
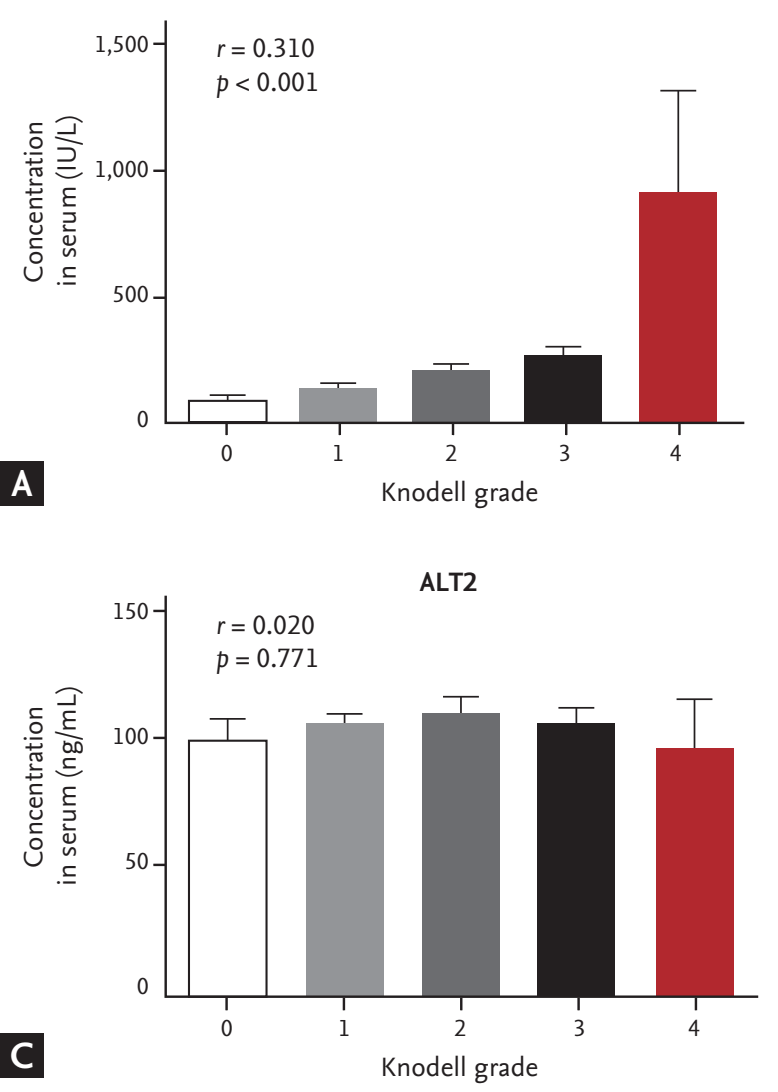

ALT1
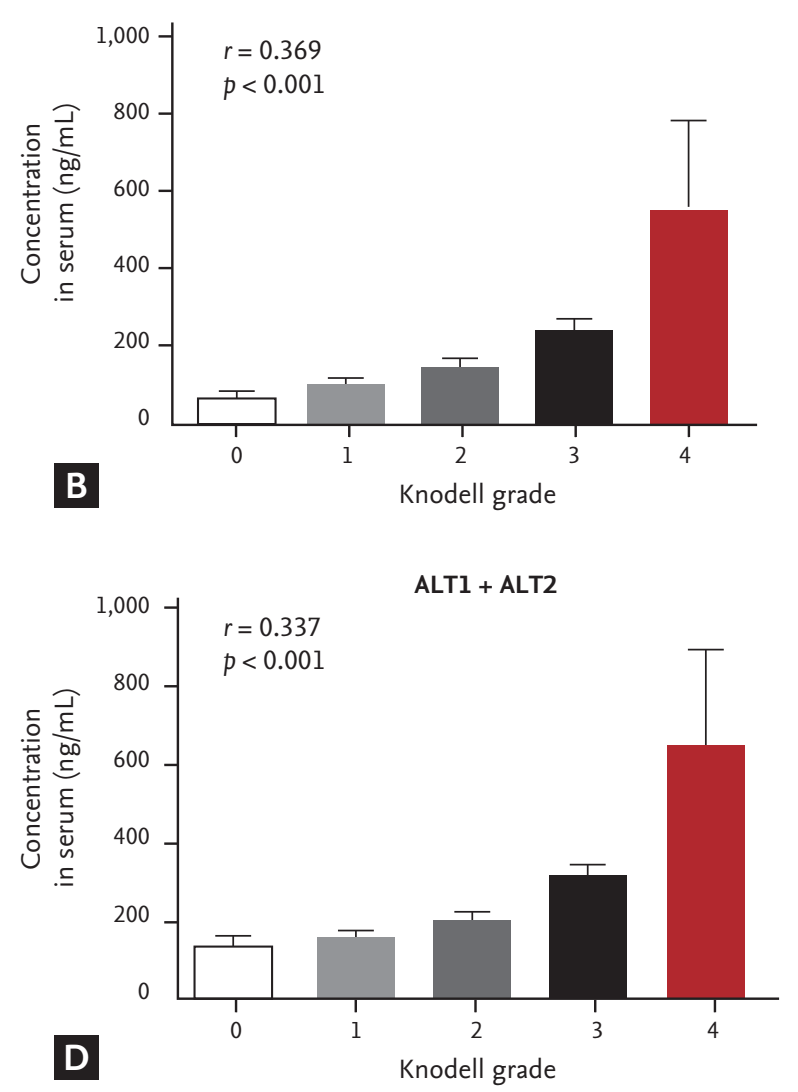

Figure 4. Distribution of alanine aminotransferase (ALT) mass concentration with Knodell grade. Serum samples were measured side by side for ALT concentration with an immunoassay. (A) Immunoassay of enzymatic ALT with Knodell grade. (B) Immunoassay of immunologic ALT1 with Knodell grade. (C) Immunoassay of immunologic ALT2 with Knodell grade. (D) Immunoassay of immunologic ALT1 + ALT2 with Knodell grade.

grades were Go (99.4), G1 (105.2), G2 (108.8), G3 (105.6), and $\mathrm{G}_{4}$ (94.6), showing no significant correlation $(p=0.771, r=$ o.020). ALT1 + ALT2 levels (ng/mL) according to inflammation grade were determined as follows: Go (139.9), $\mathrm{G}_{1}$ (159.8), G2 (208.1), G3 (316.8), and G4 (641.5), showing significant correlation $(p<0.001, r=0.337)$ and levels of $\mathrm{ALT}+\mathrm{ALT} 1$ + ALT2 were shown as Go (229.2), G1 (326.5), $\mathrm{G}_{2}$ (448.5), G3 (604.4), and $\mathrm{G}_{4}(1552.5)(p<0.001, r=0.359)$. Although ALT2 did not show significant correlation with Knodell grade of inflammation, ALT, ALT1, ALT1 + $\mathrm{ALT} 2$, and ALT + ALT1 + ALT2 levels were significantly correlated with Knodell grade of inflammation (Fig. 4).

\section{Correlation between AST and METAVIR stage}

AST levels (IU/L) according to fibrosis stage were as follows: Fo (39.6), F1 (72.6), F2 (107.6), F3 (109.4), and F4 (156.9), showing no significant correlation $(p=0.103, r=$
0.110), Levels of cAST (ng/mL) according to fibrosis stage were Fo (79.9), F1 (68.5), F2 (90.9), F3 (99.9), and F4 (102.5) $(p=0.254, r=0.077)$. The mean levels of mAST $(n g / m L)$ were observed as Fo (19.3), F1 (31.4), F2 (18.9), F3 (32.6), and $\mathrm{F}_{4}(38.8)(p=0.164, r=0.094)$, and levels of cAST $+\mathrm{mAST}$ (ng/mL) were shown as Fo (99.2), F1 (99.9), F2 (106.1), F3 (145.2), and $\mathrm{F}_{4}$ (147.6) in each fibrosis stage $(p=0.181, r=$ 0.091). The mean levels of AST, cAST, mAST, and cAST + mAST did not show correlation with the Knodell grade (Fig. 5).

\section{Correlation between AST and Knodell grade of inflammation}

Mean levels of AST (IU/L) according to Knodell inflammation grade were Go (59.8), G1 (66.7), G2 (99.6), G3 (158.8), and $\mathrm{G}_{4}$ (492.0), showing significant correlation ( $p$ $<0.001, r=0.374)$. Levels of cAST (ng/mL) according to 
AST

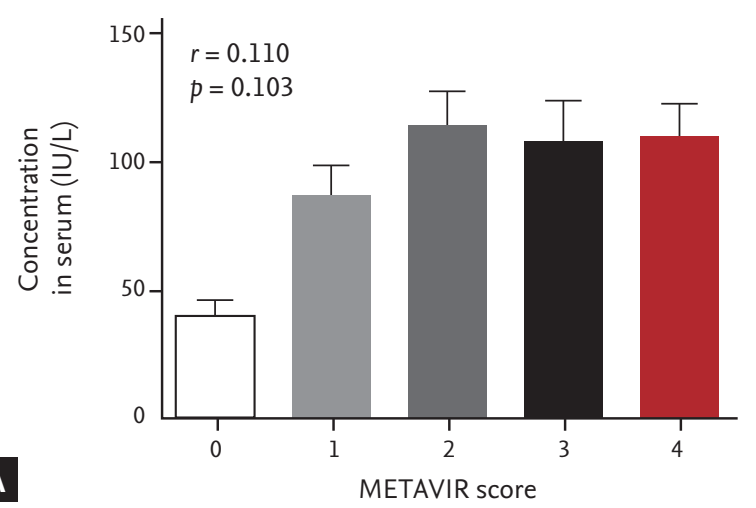

mAST

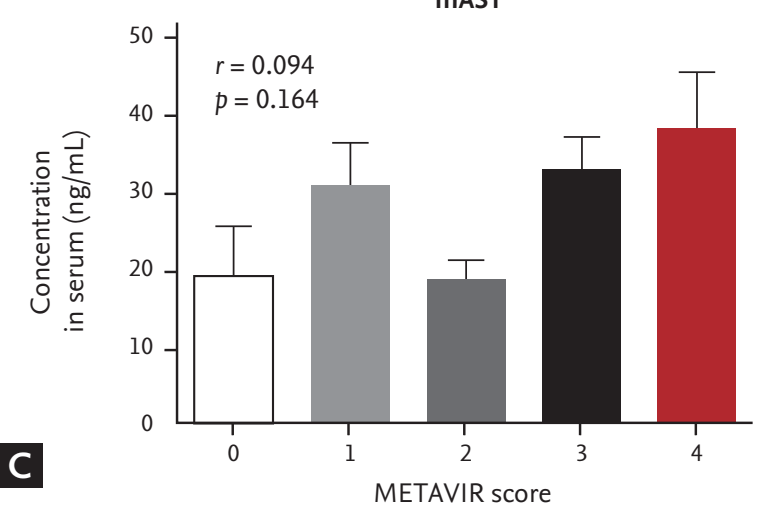

cAST
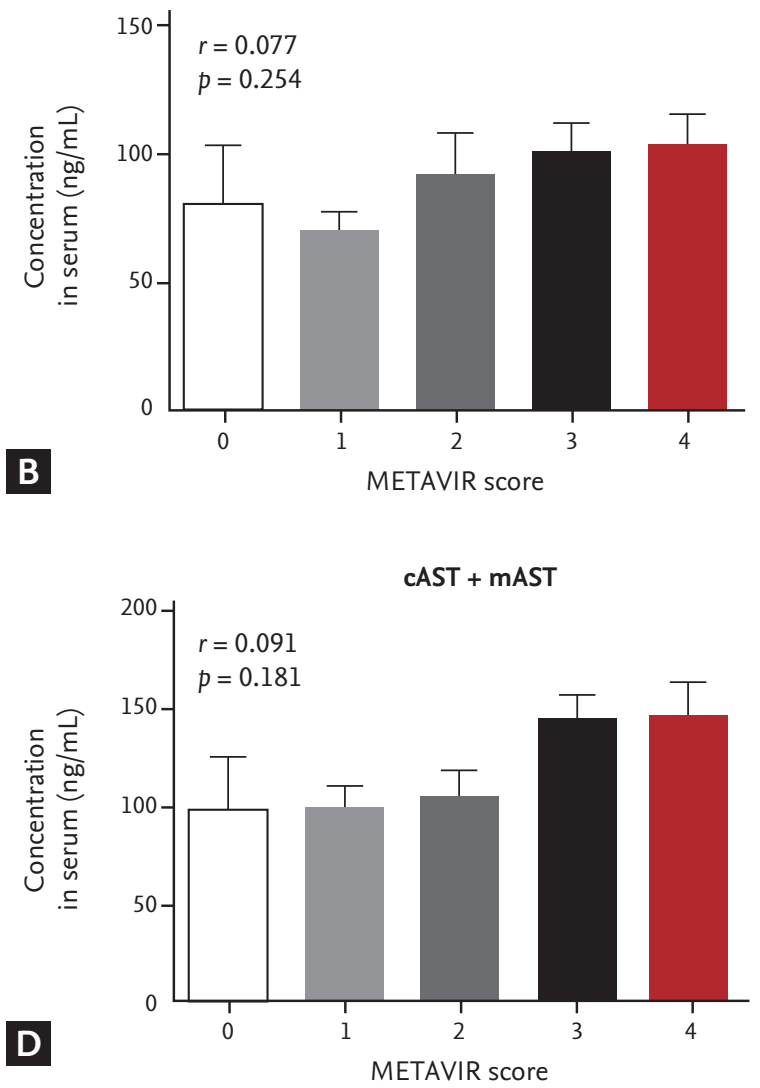

Figure 5. Distribution of aspartate aminotransferase (AST) mass concentration with METAVIR score. Serum samples were measured side by side for AST concentration with an immunoassay. (A) Immunoassay of enzymatic AST with METAVIR score. (B) Immunoassay of immunologic cytoplasmic AST (cAST) with METAVIR score. (C) Immunoassay of immunologic alanine aminotransferase 2 (ALT2) with METAVIR score. (D) Immunoassay of immunologic cAST + mitochondrial AST (mAST) with METAVIR score.

Knodell inflammation grade were Go (98.34), G1 (64.02), G2 (97.86), G3 (99.85), and $\mathrm{G}_{4}$ (79.5), showing no significant correlation $(p=0.227, r=0.082)$. Levels of mAST levels according to Knodell grade were Go (37.9), G1 (20.9), G2 (26.4), G3 (30.6), and G4 (35.6), showing no significant correlation $(p=0.477, r=0.048)$. Levels of cAST $+\mathrm{mAST}$ levels $(\mathrm{ng} / \mathrm{mL})$ according to Knodell grade were Go (136.2), G1 (84.9), G2 (124.3), G3 (130.4), and G4 (115.1), showing no significant correlation $(p=0.216, r=0.084)$. Mean levels of AST + cAST + mAST according to inflammation grade were: Go (169.04), G1 (151.62), G2 (223.85), G3 (289.23), and $\mathrm{G}_{4}$ (607.1), showing significant correlation $(p<0.001, r=0.286)$. Thus mean levels of cAST, mAST, and cAST + mAST did not show significant correlation with the Knodell grade of inflammation. However, the levels of AST and AST + cAST + mAST were significantly correlated with Knodell grade of inflammation (Fig. 6).

\section{DISCUSSION}

Although HVPG is an invasive procedure, it is known as the best predictor for the development of varices and harbinger of decompensation [21-23]. In the present study, HVPG measurement showed a positive correlation with the fibrosis stage $(r=0.439)$. Previous reports have suggested that HVPG is a better diagnostic modality than serologic biomarkers in the prediction of advanced fibrosis [24]. Garcia-Tsao et al. [25] have reported that a significant proportion of patients who did not have cirrhosis according to histological diagnosis, had HVPG > $6 \mathrm{mmHg}$. In another study, HVPG was strongly correlated with biopsy results, with HVPG $6 \mathrm{mmHg}$ demonstrating $78 \%$ and $81 \%$ accuracy in terms of sensitivity and specificity for the diagnosis of stage 1 compensated LC [26]. These results suggest that clinicians 
AST

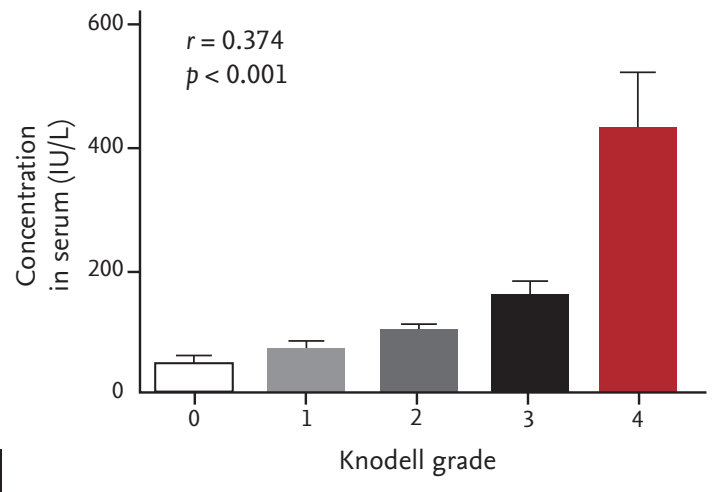

mAST

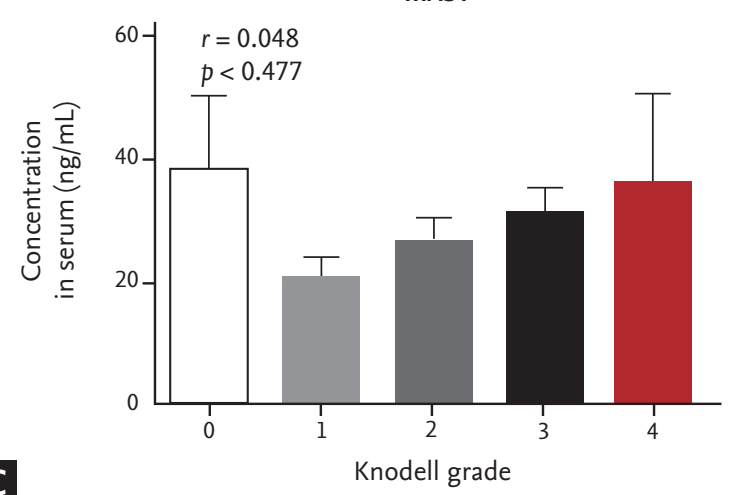

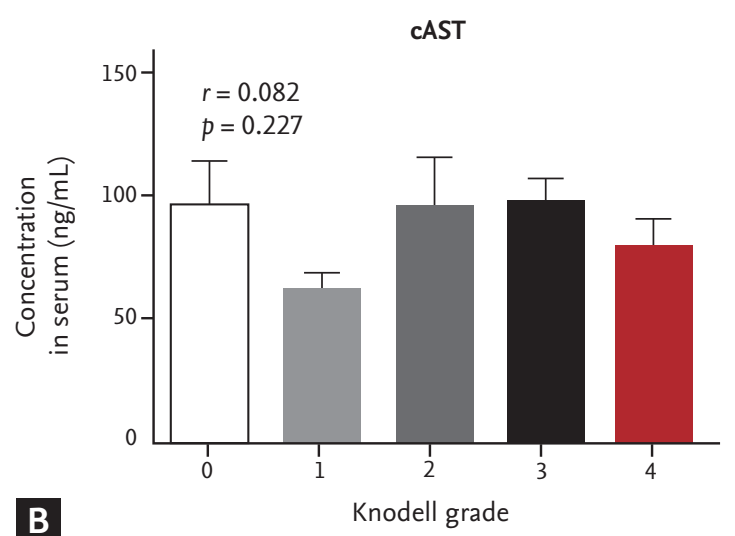

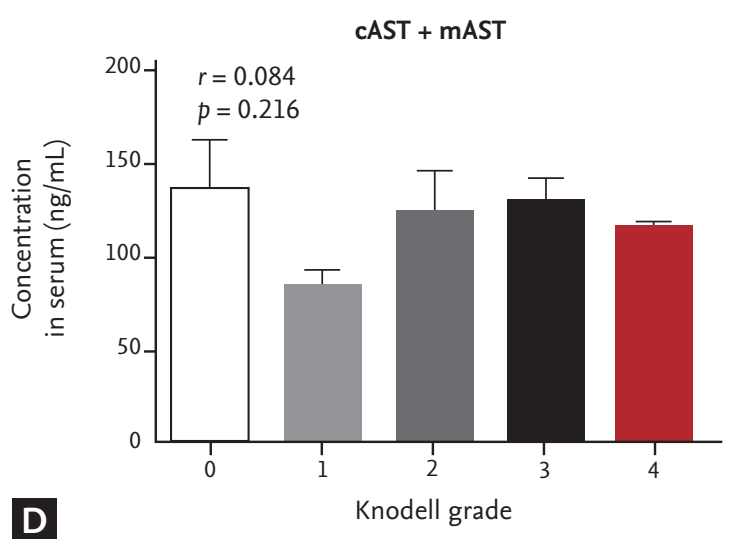

Figure 6. Distribution of aspartate aminotransferase (AST) mass concentration with Knodell grade. Serum samples were measured side by side for AST concentration with an immunoassay. (A) Immunoassay of enzymatic AST with Knodell grade. (B) Immunoassay of immunologic cytoplasmic AST (cAST) with Knodell grade. (C) Immunoassay of immunologic alanine aminotransferase 2 (ALT2) with Knodell grade. (D) Immunoassay of immunologic cAST + mitochondrial AST (mAST) with Knodell grade.

may consider performing HVPG measurement for the diagnosis of liver fibrosis in patients with CHB.

Although ALT has represented hepatocyte injury, most patients with LC have persistently normal ALT. Therefore, level of ALT has not been used as a marker for the diagnosis of chronic liver disease. In this study, measured results of ALT1 using immunologic assay showed significant correlation with fibrosis stage and inflammation grade in patients with CHB (Figs. $3 \mathrm{~B}$ and $\left.{ }_{5} \mathrm{~B}\right)$. Other studies using isoforms of ALT to analyze and diagnose of chronic liver disease or fibrosis have not been reported yet. Some previous studies demonstrated that the amount of the ALT-immunoglobulin complex is increased with the severity of liver disease [4]. Taken together, total ALT level might increase according to the progression of liver disease. Thus, the detection of ALT1 alone with its isoforms by immunoassay may be useful for the diagnosis of fibrosis and inflammation of liver disease.

Enzymatic measurements of ALT and AST were positively correlated with inflammation grade $(r=0.310$ and $r=0.374$, respectively) in patients with CHB (Figs. $4 \mathrm{~A}$ and $6 \mathrm{~A})$. This result is consistent with the findings of a previous study. Early studies have suggested that a high AST/ALT ratio may be used as a biological marker in the diagnosis of inflammation $[6,7]$. Currently, clinicians are using AST/ALT ratio for the diagnosis of specific liver disease. Wong et al. [27] have suggested that a combined algorithm involving AST to platelet ratio index (APRI) and AST/ALT could improve the accuracy in predicting advanced liver fibrosis. Therefore, enzymatic measurements of ALT and AST, according to inflammation grade, may be useful in the diagnosis of liver disease.

In most types of liver disease, serum ALT exhibits 
greater enzymatic activity than serum AST. ALT activity has been considered as a more sensitive marker of hepatocellular injury and liver disease progression than AST activity [3]. However, the development of an accurate diagnostic method is critical for early detection and proper treatment of liver disease because patients are often asymptomatic until their livers have deteriorated severely. In this study, we developed a sandwich immunoassay that uses murine monoclonal antibodies generated against human recombinant ALT1, ALT2, cAST, and $\mathrm{mAST}$ protein $[4,13]$.

Immunologic assay of ALT2, cAST, or mAST did not show correlation with the fibrosis stage and inflammation grade. Mean levels of ALT2 were negatively correlated with fibrosis stage $(r=-0.108)$. However, they showed similarity in inflammation grade $(r=0.020)$. cAST + mAST levels were positively correlated with fibrosis stage, although the correlation was not statistically significant $(r=0.107)$ (Fig. 4D). They were significantly correlated with inflammation grade $(r=0.084)$ (Fig. 6D). Therefore, detection of ALT2, cAST, mAST, and cAST + mAST using immunoassay of may not be effective for the diagnosis of liver disease.

In general, serological markers such as AST/ALT used for the diagnosis of chronic liver disease have been found to have limited diagnostic value [28]. Thus, serological markers might only be utilized as supplementary information to clinicians. Although they cannot replace needle biopsy, serological tests may provide clinicians with important information regarding staging fibrosis. They may also play a role in determining the selection and timing of treatment [29-31]. ALT1 is a good candidate for the diagnosis of liver fibrosis and inflammation.

Nowadays, non-invasive methods involving elastography, magnetic resonance elastography, fibroscan, or contrast-enhancing ultrasound are available in the diagnosis of advanced fibrosis [27,32-34]. In the near future, the incorporation of genetics, proteomics, or metabolomics might allow us to identify liver fibrosis and inflammation. Further evaluation of novel markers is required to improve the accuracy of both diagnosis and treatment.

This study suggested several significantly correlation with fibrosis stage or inflammation grade. However, this study has limitations. Because evaluation criteria of all data were only mean values, low abundances of detec- tion unit were observed in advanced fibrosis stage or inflammation grade while high abundances was observed in low stage or grade.

In conclusion, this study suggested that significantly correlated immunologic assay of ALT1 with fibrosis stage and inflammation grade might help diagnose various liver diseases in patients with CHB. Further studies are needed to understand the correct methods of using ALT and its individual isoforms for the diagnosis of liver disease in patients with CHB.

\section{KEY MESSAGE}

1. Alanine aminotransferase 1 (ALT1) measurement by utilizing sandwich enzyme-linked immunosorbent assay (ELISA) immunoassay can be useful method not only in predicting inflammation grade but also fibrosis stage in patients with chronic hepatitis B (CHB).

2. Hepatic venous pressure gradient measurement is necessary in the diagnosis of liver fibrosis in patients with CHB.

\section{Conflict of interest}

No potential conflict of interest relevant to this article was reported.

\section{Acknowledgments}

This study was funded by Hallym University Specialization Fund (HRF-S-53). This research was supported through a grant from Basic Science Research Program through the National Research Foundation of Korea (NRF) funded by the Ministry of Education, Science and Technology (NRF-2018M3A9F3020956 and NRF2018M3A9F3020942). This research was supported by Hallym University Research Fund and Hallym University Research Fund 2016 (HURF-2016-60).

\section{REFERENCES}

1. Felig P. The glucose-alanine cycle. Metabolism 1973;22:179207.

2. Daxboeck F, Gattringer R, Mustafa S, Bauer C, Assadian O. Elevated serum alanine aminotransferase (ALT) levels in 
patients with serologically verified Mycoplasma pneumoniae pneumonia. Clin Microbiol Infect 2005;11:507-510.

3. Dufour DR, Lott JA, Nolte FS, Gretch DR, Koff RS, Seeff LB. Diagnosis and monitoring of hepatic injury. I. Performance characteristics of laboratory tests. Clin Chem 2000;46:2027-2049.

4. Kim HJ, Oh SW, Kim DJ, Choi EY. Abundance of immunologically active alanine aminotransferase in sera of liver cirrhosis and hepatocellular carcinoma patients. Clin Chem 2009;55:1022-1025.

5. Kim WR, Flamm SL, Di Bisceglie AM, Bodenheimer HC; Public Policy Committee of the American Association for the Study of Liver Disease. Serum activity of alanine aminotransferase (ALT) as an indicator of health and disease. Hepatology 2008;47:1363-1370.

6. Nyblom H, Berggren U, Balldin J, Olsson R. High AST/ ALT ratio may indicate advanced alcoholic liver disease rather than heavy drinking. Alcohol Alcohol 2004;39:336339.

7. Nyblom H, Bjornsson E, Simren M, Aldenborg F, Almer S, Olsson R. The AST/ALT ratio as an indicator of cirrhosis in patients with PBC. Liver Int 2006;26:840-845.

8. Lo RC, Kim H. Histopathological evaluation of liver fibrosis and cirrhosis regression. Clin Mol Hepatol 2017;23:302-307.

9. Glinghammar B, Rafter I, Lindstrom AK, et al. Detection of the mitochondrial and catalytically active alanine aminotransferase in human tissues and plasma. Int J Mol Med 2009;23:621-631.

10. Ford GC, Eichele G, Jansonius JN. Three-dimensional structure of a pyridoxal-phosphate-dependent enzyme, mitochondrial aspartate aminotransferase. Proc Natl Acad Sci U S A 1980;77:2559-2563.

11. Stankewicz MJ, Cheng S, Martinez-Carrion M. Mitochondrial glutamate aspartate transaminase. Differential action of thiol reagents with the supernatant enzyme. Biochemistry 1971;10:2877-2884.

12. Watanabe T, Wada H. Comparative studies on the primary structure of soluble and mitochondrial glutamic oxaloacetic transaminase isozymes. I. Similar peptides isolated by cyanogen bromide cleavage. Biochem Biophys Res Commun 1971;43:1310-1317.

13. Jeong SY, Kim KJ, Kim DJ, Oh SW, Choi EY. Sandwich ELISA for measurement of cytosolic aspartate aminotransferase in sera from patients with liver diseases. Clin Chem 2003;49:826-829.
14. Leonardi F, Maria N, Villa E. Anticoagulation in cirrhosis: a new paradigm? Clin Mol Hepatol 2017;23:13-21.

15. Runyon BA; AASLD. Introduction to the revised American Association for the Study of Liver Diseases Practice Guideline management of adult patients with ascites due to cirrhosis 2012. Hepatology 2013;57:1651-1653.

16. Burroughs AK, Thalheimer U. Hepatic venous pressure gradient in 2010: optimal measurement is key. Hepatology 2010;51:1894-1896.

17. Ozkurt H, Keskiner F, Karatag O, Alkim C, Erturk SM, Basak M. Diffusion weighted MRI for hepatic fibrosis: impact of b-value. Iran J Radiol 2014;11:e3555.

18. Desmet VJ. Knodell RG, Ishak KG, et al. Formulation and application of a numerical scoring system for assessing histological activity in asymptomatic chronic active hepatitis [Hepatology 1981;1:431-435]. J Hepatol 2003;38:382-386.

19. Asselah T, Marcellin P, Bedossa P. Improving performance of liver biopsy in fibrosis assessment. J Hepatol 2014;61:193-195.

20. Klingenberg B, Agresti A. Multivariate extensions of McNemar's test. Biometrics 2006;62:921-928.

21. Groszmann RJ, Garcia-Tsao G, Bosch J, et al. Beta-blockers to prevent gastroesophageal varices in patients with cirrhosis. N Engl J Med 2005;353:2254-2261.

22. Ripoll C, Groszmann R, Garcia-Tsao G, et al. Hepatic venous pressure gradient predicts clinical decompensation in patients with compensated cirrhosis. Gastroenterology 2007;133:481-488.

23. Kim MY, Baik SK, Yea CJ, et al. Hepatic venous pressure gradient can predict the development of hepatocellular carcinoma and hyponatremia in decompensated alcoholic cirrhosis. Eur J Gastroenterol Hepatol 2009;21:12411246.

24. Kumar M, Kumar A, Hissar S, et al. Hepatic venous pressure gradient as a predictor of fibrosis in chronic liver disease because of hepatitis B virus. Liver Int 2008;28:690698.

25. Garcia-Tsao G, Friedman S, Iredale J, Pinzani M. Now there are many (stages) where before there was one: In search of a pathophysiological classification of cirrhosis. Hepatology 2010;51:1445-1449.

26. Suk KT, Kim HC, Namkung S, et al. Diagnostic accuracy of hepatic venous pressure gradient measurement in the prediction of stage 1 compensated liver cirrhosis in patients with chronic hepatitis B. Eur J Gastroenterol Hepatol 2013;25:1170-1176. 
27. Wong GL, Wong VW, Choi PC, Chan AW, Chan HL. Development of a non-invasive algorithm with transient elastography (Fibroscan) and serum test formula for advanced liver fibrosis in chronic hepatitis B. Aliment Pharmacol Ther 2010;31:1095-1103.

28. Gressner OA, Weiskirchen R, Gressner AM. Biomarkers of liver fibrosis: clinical translation of molecular pathogenesis or based on liver-dependent malfunction tests. Clin Chim Acta 2007;381:107-113.

29. Cowan ML, Rahman TM, Krishna S. Proteomic approaches in the search for biomarkers of liver fibrosis. Trends Mol Med 2010;16:171-183.

30. Paradis V. Glycomics: a new taste of cirrhosis marker. J Hepatol 2005;43:913-914.

31. Baranova A, Liotta L, Petricoin E, Younossi ZM. The role of genomics and proteomics: technologies in studying non-alcoholic fatty liver disease. Clin Liver Dis 2007;11:209-220.

32. Lee MH, Cheong JY, Um SH, et al. Comparison of surrogate serum markers and transient elastography (Fibroscan) for assessing cirrhosis in patients with chronic viral hepatitis. Dig Dis Sci 2010;55:3552-3560.

33. Huh JW, Kim YH, Kim DS, et al. Alu-derived old world monkeys exonization event and experimental validation of the LEPR gene. Mol Cells 2010;30:201-207.

34. Rustogi R, Horowitz J, Harmath C, et al. Accuracy of MR elastography and anatomic MR imaging features in the diagnosis of severe hepatic fibrosis and cirrhosis. J Magn Reson Imaging 2012;35:1356-1364. 